\title{
Genetic Association Studies in Complex Disease: Disentangling Additional Predisposing Loci from Associated Neutral Loci Using a Constrained - Permutation Approach
}

\author{
G.T. Spijker ${ }^{1}$, I.M. Nolte ${ }^{2}$, R.C. Jansen ${ }^{3}$ and G.J. Te Meerman ${ }^{1}$ \\ ${ }^{1}$ Department of Medical Genetics, University of Groningen, A. Deusinglaan 4, 9713 AW Groningen, the Netherlands \\ ${ }^{2}$ Department of Pathology and Laboratory Medicine, University of Groningen, Hanzeplein 1, 9713 GZ Groningen, the Netherlands \\ ${ }^{3}$ Groningen Bioinformatics Centre, Institute of Mathematics and Computing Science (IWI), University of Groningen, PO-box 800, \\ 9700 AV Groningen, the Netherlands
}

\section{Summary}

In the process of genetically mapping a complex disease, the question may arise whether a certain polymorphism is the only causal variant in a region. A number of methods can answer this question, but unfortunately these methods are optimal for bi-allelic loci only. We wanted to develop a method that is more suited for multi-allelic loci, such as microsatellite markers. We propose the Additional Disease Loci Test (ADLT): the alleles at an additional locus are permuted within the subsample of haplotypes that have identical alleles at the predisposing locus. The hypothesis being tested is, whether the predisposing locus is the sole factor predisposing to the trait that is in LD with the additional locus under study. We applied ADLT to simulated datasets and a published dataset on Type 1 Diabetes, genotyped for microsatellite markers in the HLA-region. The method showed the expected number of false-positive results in the absence of additional loci, but proved to be more powerful than existing methods in the presence of additional disease loci. ADLT was especially superior in datasets with less LD or with multiple predisposing alleles. We conclude that the ADLT can be useful in identifying additional disease loci.

\section{Introduction}

When a disease is mapped to a genomic region, we still face the challenge of identifying the predisposing genetic variant(s). This is mostly attempted using tests that rely on association between a specific allele and the disease. These tests assess whether the allele frequencies observed in a sample of patients deviate from the expectation, based on either relatives (e.g. parents) or unrelated people from the same population. A predisposing variant will be observed more often in patients than expected, but non-causal polymorphisms at neighbouring

*Correspondence to: Dr. G. J. te Meerman A. Deusinglaan 49713 AW Groningen the Netherlands Tel: +31-50-363 2939 Fax: +31-50-363 3113 E-mail: G. J.te.Meerman@med.rug.nl loci can show association as well. This is caused by linkage disequilibrium (LD): the phenomenon that alleles at neighbouring loci often show correlation, probably due to shared ancestry. When LD is strong, multiple loci can show comparable degrees of association, making conclusions concerning causality difficult. An additional level of complexity is present if the genomic region has more than one locus predisposing to the disease. The Human Leukocyte Antigen (HLA) region on chromosome 6 presents a clear example of these problems. This region carries $\sim 200$ genes, many of which are involved in the immune system, while LD is known to be strong. Many diseases, most notably auto-immune diseases such as Type 1 diabetes, rheumatoid arthritis, psoriasis, systemic lupus erythematosis, and multiple sclerosis, 
have shown association to this region, and many variants have been implicated (Beck \& Trowsdale, 2000).

Let us first define some vocabulary to simplify the discussion. Suppose that locus D has variants that are known or hypothesized to predispose to disease. We call this a primary locus. Suppose a set of polymorphisms in the neighborhood of D is genotyped (either functional or neutral). Some or all of these polymorphisms might be in LD with each other and/or the causal variants at locus D. We call these secondary loci.

When it is known or hypothesized that the variation at $\mathrm{D}$ is predisposing, then a number of methods are available to screen whether secondary polymorphisms additionally explain disease risk. A significant result has two possible explanations: the primary locus is not the only predisposing gene in the region, or, alternatively, the primary locus is not the true predisposing locus. They do not permit a choice between these explanations. In the following, we will briefly discuss existing methods and give some arguments as to why these are expected to perform sub-optimally. Broadly speaking, the methods fall into three categories:

1. Methods that compare allele frequencies at secondary loci within the subset of patients and controls with a specific genotype at the primary locus (Lie et al. 1999, Moghaddam et al. 1997). In addition, variants of the transmission/disequilibrium test (Lie et al. 1999) and the affected sib-pair method (Robinson et al. 1993) have been designed. Both use only parents (of affected offspring) that are homozygous for a specific allele at the primary locus. When this locus is the only predisposing locus, all secondary loci should show random transmission.

2. The so-called Haplotype Method (HM), which compares allele frequencies at the secondary locus (between patient and control haplotypes), within the subset of haplotypes that have identical alleles at the primary locus (Valdes \& Thomson, 1997; Li, 2001).

3. Disease risk modelling approaches, using multiple markers as input parameters to estimate disease risk. Examples are numerous, e.g. MASC (ClergetDarpoux et al. 1988; Valdes et al. 2001), stepwise logistic-regression (Cordell \& Clayton, 2002), log-linear modelling of haplotype effects (Rubio et al. 2002). The Conditional Extended Transmis-
sion/Disequilibrium Test (CETDT) (Koeleman et al. 2000a, 2000b), also falls in this category. This test compares risk of transmission to affected offspring for haplotypes that have identical alleles at the primary locus, but differ at the secondary locus.

Methods that use subsets of patients and controls (1 and 2) have the disadvantage that a (often substantial) part of the sample has to be discarded. Considerable numbers of patients and controls have to be screened to find sufficient numbers of subjects with the genotype of interest. Furthermore, when LD is not too strong, haplotypes that carry different alleles at the primary, known predisposing locus, might well carry the same risk allele at the second locus. The power of a method will improve when the information from the different subgroups can be combined

Disease risk modelling approaches (3) have the disadvantage of making assumptions about how the alleles at the primary locus interact. In reality, little is known about this. Estimation of this interaction is possible, but at the cost of an extra parameter with the associated increase in degrees of freedom. For this reason, it is often assumed that the alleles act independently, as in the CETDT. A significant result at a secondary locus using this kind of model might well reflect a violation of the assumed multiplicative model at the disease locus (as suggested by the simulation results below). Furthermore, the CETDT only shows the desired test properties when sample sizes are quite substantial, in the order of 500 trios for models with two alleles at the primary locus (F. Dudbridge, personal communication). For predisposing loci with many different alleles, such as HLA loci, the number of trios needed will increase even further before the test requirements are met.

Recently, Cordell \& Clayton (2002) proposed stepwise multiple logistic regression to find the set of polymorphisms that best explains disease risk. This method was shown to perform better than CETDT, as it utilizes a higher proportion of the available trios and has fewer degrees of freedom. Their implementation is currently still confined to bi-allelic loci. However, as the number of degrees of freedom will increase substantially when multi-allelic markers are tested, we expect a lower efficiency of the method. 
From the preceding survey we conclude that there still is a need for better methods: methods that combine the information from haplotypes that differ at the primary locus; perform optimally in a multi-allelic situation; and do not require assumptions about disease models.

We therefore propose an approach that is a modification of the permutation procedure for the Haplotype Method (Li, 2001). In the following, the method will be explained in detail. To assess its test characteristics, the method will be applied to simulated datasets. Finally, we show the results of the method on a real dataset: a genomic fine-screen in the HLA-region of Type 1 diabetes patients. Results are compared to HM (from which the method is derived), and to CETDT, as that is the most general approach suited to multi-allelic markers and is the most thoroughly tested.

\section{Methods and Materials}

\section{Constrained permutation}

Suppose we have a dataset of haplotypes composed of loci $\mathrm{D}$ and $\mathrm{L}$ with locus $\mathrm{D}$ a primary locus, and $\mathrm{L}$ an arbitrary secondary locus. Suppose locus D has alleles $h=$ $1, \ldots, H$. For each allele $h$ at locus D, the subgroup of haplotypes (both patient and control) carrying allele $h$ is identified, and the alleles observed at locus L are permuted within that subset of haplotypes. This procedure allows us to study the allele frequency distribution at $\mathrm{L}$ under the constraint that the association at $\mathrm{D}$ is fixed. The permutation procedure implicitly assumes random mating, but only within the subsets of haplotypes that have identical alleles at the primary locus.

\section{Additional disease locus test}

The secondary locus L can have multiple alleles $i$ occurring at different frequencies within patients and controls. A chi-square statistic is computed on the $2 \mathrm{x} I$ contingency table of allele frequencies at locus $\mathrm{L}$, $\mathrm{X}^{2}=\sum_{i=1}^{I} \sum_{j=p, c} \frac{\left(O_{i j}-E_{i j}\right)^{2}}{E_{i j}}$,

where $j$ designates the subgroup of haplotypes (patient or control), $O_{i j}\left(E_{i j}\right)$ is the observed (expected) number of haplotypes carrying allele $i$ in subgroup $j . E_{i j}$ is computed under the null-hypothesis that there is no association, i.e. the frequency of allele $i$ is assumed to be equal in patients and controls for all $i$. The chi-square is computed for the original sample of haplotypes and also for each permuted set of haplotypes; it has $I-1 \mathrm{de}-$ grees of freedom. Note that the distribution of $X^{2}$ in the permutations can deviate markedly from the neutral expectation, due to possible LD between D and L. For this reason, the proportion of permutations that have a $X^{2}$ equal or larger than in the original sample serves as an empirical p-value. In the computation all alleles with expected values below 4 are pooled into one group.

In the simulations presented in this study we used trios consisting of an affected patient with both parents. This design enables the derivation of parental phase: the alleles in a patient that are inherited from the same parent are on the same haplotype. The alleles that are not transmitted to the patient form the control haplotypes (Falk \& Rubinstein, 1987). In practice, there is always some missing information. Permutation of incomplete haplotypes increases the variance of the test statistic, leading to a conservative test. Therefore, we performed the permutations for each secondary locus in turn, using only completely known two-locus haplotypes.

In the following, we will validate the Additional Disease Locus Test (ADLT) on several simulated samples, together with HM and CETDT.

\section{HM - Li variant}

Li proposed a different test statistic ( $\mathrm{Li}, 2001)$ : write $\mathrm{X}_{L, h}^{2}=\sum_{i=1}^{I} \sum_{j=p, c} \frac{\left(O_{i j}-E_{i j}\right)^{2}}{E_{i j}}$ for the chi-square statistic at locus $\mathrm{L}$ for the subgroup of individuals carrying allele $h$ at $\mathrm{D}$, and define $T_{L}=\max _{h} \frac{\left(X_{L, h}^{2}-v_{L, h}\right)}{\sqrt{2 v_{L, h}}}$ at the maximum over all subgroups, then Li's test statistic is the max $T_{L}$ over all secondary loci $\mathrm{L}$ under study; $v_{L, h}$ is the number of degrees of freedom. Standardization is carried out, so that under the null-hypothesis $X^{2}$ approximately follows a standard normal distribution. This is necessary for the comparison that is made in the function "max," as the subgroups of haplotypes $h$ and different loci can have contingency tables with different numbers of degrees of freedom. Comparing only the maximum $\mathrm{X}^{2}$ over all loci $\mathrm{L}$ is an efficient way of correcting for the testing at multiple loci. 


\section{Conditional extended TDT}

As a comparison, CETDT was calculated on the same simulated datasets, using the UNPHASED program set, version 2.21 (F. Dudbridge, 2003), compiled using GCC under CYGWIN.

\section{Simulated datasets}

To test the properties of these methods, we simulated datasets representing trios genotyped for some multiallelic markers. The basic simulation procedure was as follows: random allele frequency tables are generated with a maximum of 10 alleles and a heterozygosity of about 0.8 . Using these allele frequencies, four haplotypes are simulated representing two parent genotypes. LD between the loci is introduced by increasing the frequency of an allele at the secondary locus, depending on the allele chosen at the primary locus. One random haplotype of each parent is transmitted to the child, with 0.01 chance of recombination between the loci. The child has a certain chance of being affected depending on its genotype. The risk for a person without predisposing alleles is set to 0.001 . Each predisposing allele multiplies that risk with an allele specific relative risk (RR), representing a multiplicative model. Unless otherwise specified a RR of 4 is used. A draw from the random generator decides, in combination with the individual risk, whether the child indeed is deemed affected, in which case the trio is saved to file. This process is repeated until the desired sample size is reached. Resulting LD was assessed by multi-allelic D' (Hedrick, 1987), computed on the sample of control haplotypes. The simulated datasets were analyzed only using phase information that could be deduced from the pedigree.

\section{False-positive rate}

To investigate the characteristics of the methods under the null hypothesis, datasets with two loci D and L were simulated, of which only the first locus influenced disease risk. The results of the methods achieved at the second locus were assessed; the first locus was supposed to be locus D. Unless otherwise specified, the first locus was simulated to have one predisposing allele with a frequency of 0.13 . This was carried out for models with a total of 2, 3 and 8 alleles at the first locus, resulting in a heterozygosity of $0.23,0.56$ and 0.73 respectively. 1000 replicates were simulated for each model and each sample size of 100, 200 and 400 trios.

The sensitivity of the methods to deviations from a multiplicative model was assessed by simulating a model with two predisposing alleles rather than one (frequency of 0.05 and 0.08 ), each with RR of 1.5; when present heterozygously the disease risk was additionally increased 8 times. The sensitivity of the methods to missing genotypes was tested by setting a fixed percentage of genotypes at each locus as unknown.

The fraction of false positive results was computed for 2 levels of alpha: 0.05 and 0.001 . A binomial distribution can be used to test whether the observed numbers of significant results deviate from expected. The number of false positive results is significantly different from alpha $=0.05$ when more than 63 or less than 38 out of 1000 replicates have a $\mathrm{p}$-value below 0.05 . At alpha $=$ 0.001 the results are significantly different from alpha, when more than 3 out of 1000 replicates have a $p$-value below 0.001 .

\section{Conditioning at a wrong locus}

We also wanted to investigate the influence of misspecification of the primary locus. For this purpose, datasets were simulated with a third locus, in strong LD (resulting $\mathrm{D}^{\prime} \approx 0.85$ ) with the disease locus $\mathrm{D}$. The datasets were analyzed conditioning on this third locus, while testing both the true disease locus D and a neutral locus L.

\section{Power}

To assess the power of the methods, one allele at the second locus additionally modified disease risk. The first locus had in all cases one predisposing allele (freq 0.13) with an RR of 4 . The effect of the second locus was independent of the first locus. The frequency of the predisposing allele at the second locus was set to about 0.1 . The power was assessed at sample sizes of 100, 200 and 400 trios using 100 replicates. In the dominant model, the risk was multiplied 8 times when at least one predisposing allele was present at the second locus. In the 
recessive model, the risk was multiplied 8 times when two predisposing alleles were present.

To investigate the influence of LD on the test characteristics datasets were simulated for different levels of LD. To investigate the influence of missing genotypes, $5 \%, 10 \%$ and $20 \%$ of the genotypes at each locus were set to unknown. The influence of multiple predisposing alleles was assessed by simulating datasets with either two predisposing alleles (freq 0.05 and 0.08 ) at the primary locus and one predisposing allele (freq 0.1) at the second locus, or one predisposing allele (freq 0.13) at the primary locus and two predisposing alleles at the second locus (freq 0.1).

\section{Type 1 diabetes dataset}

We re-analyzed the dataset with Type 1 diabetes that is publicly available (see electronic references), to further compare the applicable methods. The dataset consists of 353 pedigrees from the Warren 1 repository, consisting of two parents and two affected offspring (Bain et al 1990). These were typed for 5 HLA loci (A, B, C, DQB1 and DRB1) and 28 polymorphic microsatellites in the HLA-region. The data were analyzed by Herr et al. (2000), and details can be found there. Among other results, they reported an association with D6S2223, which was significant when conditioning for the effects of $H L A-D Q B 1$ and $-D R B 1$ (p-value without multiple testing correction $=0.0018)$.
We used a subset of the data for analysis: pedigrees were selected with both parents and at least one child fully typed for DQB1 and DRB1. When both children were fully typed, one was chosen at random. 31 families were excluded, as one of the parents was also affected by Type 1 diabetes. The resulting dataset consisted of 239 trios. Empirical p-values were determined for ADLT and HM using $10^{7}$ permutations. For the HM both the global p-value and the results for the individual markers are reported. We used 0.001 (equivalent to $\left.-\log _{10}(\mathrm{p})=3\right)$ as the threshold to label results as significant. Note that, as TDT is a valid test for linkage in multiplex families, CETDT would have higher power when both affected sibs were included.

\section{Results}

\section{Simulation data}

\section{False-positive rate}

The fractions of false positive results are shown in Tables 1 and 2. Our simulation of LD influences the allele frequency at the neutral locus; this causes the heterozygosity at the neutral locus to be higher when the number of alleles at the predisposing locus increases. The ADLT and HM have fractions of false positive results within the expected range. CETDT has too many false positive results in the models with multiple alleles at the primary locus. In the model that deviates from the

Table 1 fraction of false positive results

\begin{tabular}{|c|c|c|c|c|c|c|c|c|}
\hline $\begin{array}{l}\text { Model at first } \\
\text { locus }\end{array}$ & $\begin{array}{l}\text { Hetero- } \\
\text { zygosity }\end{array}$ & $\begin{array}{l}\text { Sample } \\
\text { Size }\end{array}$ & $\begin{array}{l}\text { ADLT } \\
0.05\end{array}$ & 0.001 & $\begin{array}{l}\mathrm{HM} \\
0.05\end{array}$ & 0.001 & $\begin{array}{l}\text { CETDT } \\
0.05\end{array}$ & 0.001 \\
\hline \multirow[t]{3}{*}{2 alleles at primary locus } & \multirow[t]{3}{*}{0.70} & 100 & 0.060 & 0.002 & 0.061 & 0 & 0.059 & 0.001 \\
\hline & & 200 & 0.042 & 0.002 & 0.045 & 0 & 0.074 & 0 \\
\hline & & 400 & 0.048 & 0 & 0.042 & 0 & 0.063 & 0 \\
\hline \multirow[t]{3}{*}{3 alleles at primary locus } & \multirow[t]{3}{*}{0.77} & 100 & 0.052 & 0 & 0.046 & 0.001 & 0.098 & 0.002 \\
\hline & & 200 & 0.042 & 0 & 0.043 & 0.001 & 0.099 & 0.004 \\
\hline & & 400 & 0.040 & 0.001 & 0.042 & 0.001 & 0.076 & 0.001 \\
\hline \multirow[t]{3}{*}{8 alleles at primary locus } & \multirow[t]{3}{*}{0.81} & 100 & 0.039 & 0 & 0.044 & 0.001 & 0.171 & 0.001 \\
\hline & & 200 & 0.046 & 0 & 0.041 & 0 & 0.144 & 0.002 \\
\hline & & 400 & 0.041 & 0.001 & 0.040 & 0.001 & 0.129 & 0.003 \\
\hline \multirow{3}{*}{$\begin{array}{l}8 \text { alleles at primary locus; } \\
\text { not multipicative }\end{array}$} & \multirow[t]{3}{*}{0.80} & 100 & 0.042 & 0 & 0.051 & 0.003 & 0.183 & 0.004 \\
\hline & & 200 & 0.056 & 0.001 & 0.058 & 0 & 0.176 & 0.004 \\
\hline & & 400 & 0.055 & 0 & 0.046 & 0.001 & 0.175 & 0.009 \\
\hline
\end{tabular}

Fraction of false positive results in 1000 replications at alpha $=0.05$ and alpha $=0.001$. Fractions of false positive results that differ significantly from expected are shown in bold. Models are further described in the text. Resulting $\mathrm{D}^{\prime} \approx 0.5$.

Heterozygosity: median heterozygosity of the non-predisposing second locus. Samplesize: number of trio's used. 


\begin{tabular}{llllllll}
\hline Percentage & Sample & ADLT & \multicolumn{3}{c}{ HM } & \multicolumn{3}{c}{ CETDT } \\
unknown genotypes & size & 0.05 & 0.001 & 0.05 & 0.001 & 0.05 & 0.001 \\
\hline No unknown & 100 & 0.039 & 0 & 0.044 & 0.001 & $\mathbf{0 . 1 7 1}$ & 0.001 \\
& 200 & 0.046 & 0 & 0.041 & 0 & $\mathbf{0 . 1 4 4}$ & 0.002 \\
& 400 & 0.041 & 0.001 & 0.04 & 0.001 & $\mathbf{0 . 1 2 9}$ & 0.003 \\
5\% unknown & 100 & 0.053 & 0.003 & 0.048 & 0 & $\mathbf{0 . 1 7 6}$ & 0.002 \\
& 200 & 0.046 & 0 & 0.042 & 0 & $\mathbf{0 . 1 2 8}$ & $\mathbf{0 . 0 0 4}$ \\
& 400 & $\mathbf{0 . 0 3 7}$ & 0.001 & 0.038 & 0.001 & $\mathbf{0 . 1 2 0}$ & 0.001 \\
10\% unknown & 100 & 0.048 & 0.002 & 0.051 & 0.001 & $\mathbf{0 . 1 8 9}$ & $\mathbf{0 . 0 0 8}$ \\
& 200 & 0.048 & 0.002 & 0.043 & 0 & $\mathbf{0 . 1 4 7}$ & $\mathbf{0 . 0 0 5}$ \\
& 400 & 0.047 & 0 & 0.045 & 0 & $\mathbf{0 . 1 3 5}$ & 0.002 \\
20\% unknown & 100 & 0.041 & 0 & 0.044 & 0.003 & $\mathbf{0 . 1 7 4}$ & 0.003 \\
& 200 & 0.051 & 0 & 0.047 & 0.002 & $\mathbf{0 . 1 5 6}$ & 0.003 \\
& 400 & 0.044 & 0.001 & 0.062 & 0.003 & $\mathbf{0 . 1 4 9}$ & $\mathbf{0 . 0 0 7}$ \\
\hline
\end{tabular}

Fraction of false positive results in 1000 replications at alpha $=0.05$ and alpha $=0.001$. Fractions that differ significantly from expected are shown in bold. Resulting $D^{\prime} \approx 0.5$. Models are further described in the text.

\begin{tabular}{llllllll}
\hline Results at locus & Sample & ADLT & \multicolumn{3}{c}{ HM } & \multicolumn{3}{c}{ CETDT } \\
& Size & 0.05 & 0.001 & 0.05 & 0.001 & 0.05 & 0.001 \\
\hline True predisposing locus & 100 & 0.854 & 0.335 & 0.282 & 0.044 & 0.544 & 0.070 \\
& 200 & 0.986 & 0.781 & 0.748 & 0.288 & 0.810 & 0.240 \\
A neutral locus & 400 & 1.000 & 0.994 & 0.970 & 0.723 & 0.969 & 0.712 \\
& 100 & 0.148 & 0.008 & 0.072 & 0.002 & 0.342 & 0.022 \\
& 200 & 0.257 & 0.013 & 0.084 & 0.003 & 0.370 & 0.024 \\
& 400 & 0.427 & 0.058 & 0.165 & 0.013 & 0.453 & 0.049 \\
\hline
\end{tabular}

Fraction of significant results in 1000 replications at alpha $=0.05$ and alpha $=$ 0.001 , when conditioning at another locus than the true predisposing locus. Only 1 predisposing allele (freq 0.13 ) at the true primary locus, with $\mathrm{RR}=4$. Resulting LD between conditioning locus and true locus $\mathrm{D}^{\prime} \approx 0.85$; between neutral locus and true locus, and between neutral locus and conditioning locus $\mathrm{D}^{\prime} \approx 0.5$.
Table 2 Effect of missing genotypes on false positive results
Table 3 Fraction of significant results, while conditioning at a wrong locus multiplicative model, the number of false positive results is further increased for CETDT, while the ADLT and HM still have false positive results within the expected range.

The influence of missing genotypes is shown in Table 2. The ADLT and HM still have fractions of false positive results within the expected range, while CETDT exhibits an increased fraction of false positive results.

\section{Conditioning at a wrong locus}

The fraction of significant results in datasets in which the wrong locus is used for conditioning, rather than the true predisposing locus, is shown in Table 3. ADLT has the highest power when the true locus is included in the dataset. At a neutral locus, the fraction of significant results is much lower. CETDT has more results that are significant at lower sample sizes.

\section{Power}

Results for a number of disease models are shown in Tables 4 to 7. HM has the lowest power in all simulated data sets. ADLT has slightly more power than CETDT, particularly for models with more predisposing alleles at the primary locus and less LD. Decreasing levels of LD between the loci increases power for all methods, but ADLT seems to gain the most power. Missing genotypes decrease the power of CETDT and ADLT in a similar way.

\section{Type 1 diabetes dataset}

The results for the selection of Type 1 diabetes patients are presented in Table 8. When calculating an 


\begin{tabular}{lllll}
\hline Model at second locus & Sample size & ADLT & HM & CETDT \\
\hline $\mathrm{RR}=4$ & 100 & 0.42 & 0.18 & 0.30 \\
& 200 & 0.78 & 0.37 & 0.70 \\
$\mathrm{RR}=2$ & 400 & 0.98 & 0.81 & 0.93 \\
& 100 & 0.02 & 0.01 & 0.02 \\
$\mathrm{RR}=4$ with recessive effect & 200 & 0.07 & 0.02 & 0.05 \\
& 400 & 0.19 & 0.11 & 0.16 \\
$\mathrm{RR}=4$ with dominant effect & 100 & 0.04 & 0 & 0.01 \\
& 200 & 0.05 & 0.01 & 0.03 \\
& 400 & 0.19 & 0.11 & 0.15 \\
& 200 & 0.16 & 0.04 & 0.16 \\
& 400 & 0.42 & 0.12 & 0.40 \\
\hline
\end{tabular}

Power at alpha $=0.001$ based on 100 replicates per model and sample size. Resulting $D^{\prime} \approx 0.45$. Models are further described in the text.

\begin{tabular}{|c|c|c|c|c|}
\hline LD & $\begin{array}{l}\text { Sample } \\
\text { size }\end{array}$ & ADLT & $\mathrm{HM}$ & CETDT \\
\hline \multirow[t]{3}{*}{ No } & 100 & 0.85 & 0.17 & 0.38 \\
\hline & 200 & 1.00 & 0.42 & 0.87 \\
\hline & 400 & 1.00 & 0.98 & 1.00 \\
\hline \multirow[t]{3}{*}{ Weak $\quad \mathrm{D}^{\prime} \approx 0.2$} & 100 & 0.75 & 0.14 & 0.34 \\
\hline & 200 & 0.99 & 0.45 & 0.82 \\
\hline & 400 & 1.00 & 0.93 & 1.00 \\
\hline \multirow[t]{3}{*}{ Moderate $\quad \mathrm{D}^{\prime} \approx 0.3$} & 100 & 0.46 & 0.13 & 0.33 \\
\hline & 200 & 0.89 & 0.33 & 0.73 \\
\hline & 400 & 1.00 & 0.86 & 1.00 \\
\hline \multirow[t]{3}{*}{ Strong $\quad \mathrm{D}^{\prime} \approx 0.6$} & 100 & 0.31 & 0.16 & 0.16 \\
\hline & 200 & 0.52 & 0.38 & 0.49 \\
\hline & 400 & 0.85 & 0.62 & 0.85 \\
\hline
\end{tabular}

Power at alpha $=0.001$ based on 100 replicates per model and sample size.

Models are further described in the text.

(unconditional) ETDT (Sham \& Curtis, 1995) for 33 markers, 15 of the markers have a $-\log _{10}$ ( $\mathrm{p}$-value) larger than 3 (equivalent to a $\mathrm{p}$-value of 0.001). The most significant p-values are seen at $H L A-D Q B 1$ and $H L A$ $D R B 1$; both are roughly equally significant.

The overall test of the HM rejects the hypothesis that either $D Q B 1$ or $D R B 1$ are the sole predisposing loci $(\mathrm{p}-$ value resp. $<1 \mathrm{e}-7$ and 1e-7), but the hypothesis that the combination of DQB1 and DRB1 are the predisposing factors is not rejected ( $\mathrm{p}$-value $=0.144$ ).

When conditioning at locus $D Q B 1$, all methods yield highly significant results at DRB1. In this case, the ADLT shows an additional significant result at the marker D6S258, with $-\log _{10}(\mathrm{p})=4.27$. The HM has an additional significant result at C1-2-A $\left(-\log _{10}(\mathrm{p})=\right.$ 3.93).
When conditioning on locus DRB1 ADLT has equally significant results at two loci: $D Q B 1$ and D6S2444, and therefore cannot distinguish between these two. CETDT has a significant result only for $D Q B 1$, although much less extreme. The HM also shows a significant result at $H L A-D Q B 1\left(-\log _{10}(\mathrm{p})=\right.$ 7), but an additional significant association is seen at TNF-e $\left(-\log _{10}(\mathrm{p})=3.62\right)$. Four other loci near that locus show near significant results $\left(-\log _{10}\right.$ (p) over 2).

When conditioning on both $H L A-D Q B 1$ and $D R B 1$, no method yields significant results for other loci. Results approaching significance were seen for ADLT (3 markers with a p-value smaller than 0.02). These results suggest that the effects of either DQB1 or DRB1 cannot explain the risk for Type 1 diabetes independently, 


\begin{tabular}{|c|c|c|c|c|}
\hline Model at second locus & Sample Size & ADLT & HM & CETDT \\
\hline \multirow[t]{3}{*}{$\mathrm{RR}=4 \quad \mathrm{D}^{\prime} \approx 0.45$} & 100 & 0.45 & 0.11 & 0.25 \\
\hline & 200 & 0.85 & 0.44 & 0.64 \\
\hline & 400 & 0.99 & 0.83 & 0.98 \\
\hline \multirow[t]{3}{*}{$\mathrm{RR}=2 \quad \mathrm{D}^{\prime} \approx 0.45$} & 100 & 0.02 & 0.01 & 0.02 \\
\hline & 200 & 0.07 & 0.02 & 0.05 \\
\hline & 400 & 0.19 & 0.06 & 0.15 \\
\hline \multirow[t]{3}{*}{$\mathrm{RR}=2 \quad \mathrm{D}^{\prime} \approx 0.3$} & 100 & 0.55 & 0.11 & 0.30 \\
\hline & 200 & 0.93 & 0.52 & 0.79 \\
\hline & 400 & 1.00 & 0.83 & 0.99 \\
\hline \multicolumn{5}{|c|}{ B: Two predisposing alleles at second locus, one at primary locus } \\
\hline \multirow[t]{3}{*}{$\mathrm{RR}=4 \quad \mathrm{D}^{\prime} \approx 0.45$} & 100 & 0.66 & 0.16 & 0.41 \\
\hline & 200 & 0.99 & 0.62 & 0.91 \\
\hline & 400 & 1.00 & 0.94 & 1.00 \\
\hline \multirow[t]{3}{*}{$\mathrm{RR}=2 \quad \mathrm{D}^{\prime} \approx 0.45$} & 100 & 0.03 & 0 & 0.03 \\
\hline & 200 & 0.13 & 0.06 & 0.15 \\
\hline & 400 & 0.34 & 0.19 & 0.42 \\
\hline \multirow[t]{3}{*}{$\mathrm{RR}=2 \quad \mathrm{D}^{\prime} \approx 0.3$} & 100 & 0.07 & 0 & 0.09 \\
\hline & 200 & 0.22 & 0.02 & 0.10 \\
\hline & 400 & 0.54 & 0.16 & 0.43 \\
\hline
\end{tabular}

Table 6 Power in models with multiple predisposing alleles A: Two predisposing alleles at primary locus, one at second locus

Power at alpha $=0.001$ based on 100 replicates per model and sample size. Models are further described in the text.

\begin{tabular}{lllll}
\hline Sample size & Missing genotypes & ADLT & HM & CETDT \\
\hline 100 & $0 \%$ & 0.42 & 0.18 & 0.30 \\
& $5 \%$ & 0.38 & 0.15 & 0.31 \\
& $10 \%$ & 0.27 & 0.12 & 0.15 \\
200 & $20 \%$ & 0.13 & 0.05 & 0.06 \\
& $0 \%$ & 0.78 & 0.37 & 0.70 \\
& $5 \%$ & 0.74 & 0.36 & 0.49 \\
& $10 \%$ & 0.63 & 0.28 & 0.34 \\
400 & $20 \%$ & 0.39 & 0.16 & 0.22 \\
& $0 \%$ & 0.98 & 0.81 & 0.93 \\
& $5 \%$ & 0.99 & 0.69 & 0.90 \\
& $10 \%$ & 0.93 & 0.55 & 0.82 \\
& $20 \%$ & 0.68 & 0.34 & 0.45 \\
\hline
\end{tabular}

Table 7 Influence of missing genotypes on power

Power at alpha $=0.001$ based on 100 replicates per model and sample size. Models are further described in the text

but there is little evidence for the influence of additional loci.

\section{Discussion}

We developed the Additional Disease Locus Test (ADLT) to test whether a certain locus explains all the disease risk in a genomic region. At secondary loci it combines the information from haplotypes that have different alleles at locus $\mathrm{D}$, without making specific assumptions about the disease model.

We have shown ADLT to have the expected distribution in the absence of an effect at secondary loci; thus this method is indeed able to correct for LD effects of known predisposing loci, also when the data is incomplete. ADLT showed better power than existing methods, especially in datasets with multiple predisposing alleles at locus D, and at low levels of LD between the markers.

\section{ADLT compared to other methods}

When compared to HM, ADLT showed markedly higher power on simulated datasets, due to combining information of haplotypes with different alleles at locus 


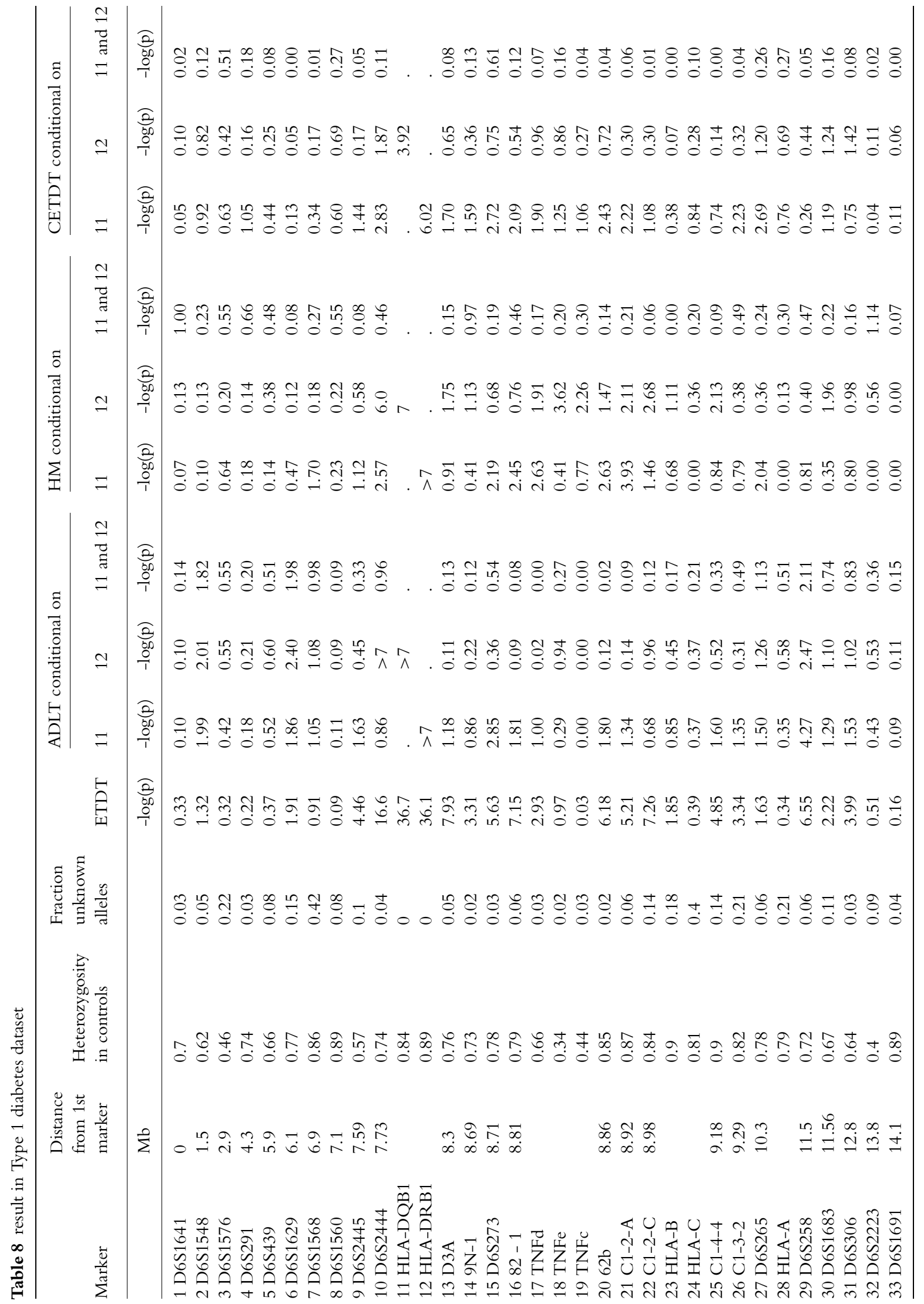


D. The difference was less obvious in the results with the diabetes dataset, probably due to a very high level of LD between DQB1 and DRB1.

The ADLT and HM share several features that are advantageous over CETDT. The first two methods do not explicitly assume disease models, as is done by CETDT. Thus, the number of parameters to be estimated is small, which makes them valid for smaller sample sizes than CETDT.

The large number of parameters that are estimated is indeed the major drawback of CETDT. The assumption of a multiplicative disease model is made, in order to reduce the number of parameters, but not on biological grounds. As suggested in the introduction, deviations from the multiplicative model might cause false positive results; the simulation results support this view (Table 1, last model).

However, with many alleles at the predisposing locus the number of parameters to be estimated is still high, despite the multiplicative disease model. Therefore, many trios are required before the desired asymptotic test properties are reached. In the simulations, CETDT produced too many false positive results even for samples of 400 trios. Pooling of rare haplotypes might improve this, but most haplotypes of highly polymorphic markers are rare. For example, when conditioning on HLA-DQB1 in the Type 1 diabetes dataset, the haplotypes with a parental frequency of over $5 \%$ comprise only $48 \%$ of the haplotype counts. When conditioning at both $D Q B 1$ and $D R B 1$, this is only $26 \%$. This makes pooling of rare haplotypes quite inefficient.

An additional advantage of ADLT over CETDT is that it can be applied to trios consisting of the patient with their spouse and child as well. Such a design is disadvantageous from a methodological point of view, as it is sensitive to population stratification and nonrandom mating. However, it might still be useful for late onset diseases, as it is often difficult to enroll sufficient numbers of traditional trios.

\section{Possible limitations}

Earlier, the use of family based control alleles was shown to be sensitive to population stratification, as the test could become somewhat conservative (Ewens \& Spielman 1995). The same will apply for ADLT, al-

(C) University College London 2004 though to a lesser degree, as the permutations are constrained by the alleles observed at the primary locus.

The ADLT procedure does not account for the specific combination of two haplotypes that form a person's genotype; both haplotypes are permuted independently. One could expect this to lead to false positive results when there is a strong deviation from the multiplicative model. In our simulations (Table 1, last model) we have shown that this is not the case, while CETDT shows an increase of false positive results. Nevertheless, a variant of ADLT could be designed: permutation of transmission of haplotypes within a parent could be constrained by the transmission-nontransmission table at the predisposing locus. We expect this only to work in situations with few alleles at the primary locus or with large datasets, because otherwise many genotypes are observed only rarely. It is also possible to design a comparable constrained permutation procedure to test unphased genotypes, e.g. case-control studies. We are currently developing these extensions.

The Pearson chi-square test that we use is relatively insensitive when only one allele at a locus differs in frequency between patients and controls. When using microsatellite markers at secondary loci, this is only a minor problem. As it is unlikely that these markers are themselves predisposing to disease, we expect them only to be in some degree of LD with the causal mutation, which makes it likely that multiple alleles will show differences in frequency between patients and controls. Note that the presented simulations mostly had a predisposing effect in only one allele, and thus already represent this unfortunate situation.

One might question the validity of using only one patient from a sib pair design, ignoring the multiplex character of the families. In general, this will lead to an increased number of risk alleles in the non-transmitted haplotypes, thereby decreasing the power. This kind of approach cannot be used to reliably estimate the strength of the relative risks, but significant differences between 'case' and 'control' allele frequencies are still evidence for the presence of an effect at secondary loci.

The threshold value of alpha $=0.001$, seems a cautious choice, as a Bonferroni correction for 32 markers at 0.001 gives an equivalent $\mathrm{p}$-value of $1-(1-\mathrm{alpha})^{32}$ $\approx 0.03$. Bonferroni correction is known to be conservative for independent tests. In this situation, tests are

Annals of Human Genetics (2005) 69,90-101 
correlated due to strong LD between the markers, thus reducing the actual number of independent tests. ADLT and HM seem to perform better than CETDT on this dataset, as they have more significant results.

\section{General limitation}

An important point to note, that holds for all the methods discussed, is the following: the correction for the effect of a primary locus is only complete when the observed variation is a true causal polymorphism or in complete LD with it. In general, it cannot be concluded on statistical grounds whether a polymorphism is causal. Therefore, in the absence of additional external evidence for the involvement of the observed variation, there is always the possibility that the effect at the secondary locus is an artifact due to incomplete correction of the effect at the primary locus, and that the primary locus is not the true causal variation (Cordell \& Clayton, 2002; Li, 2001).

In conclusion, we think that the ADLT can be useful in association studies of complex disease, to distinguish between additional disease loci and apparent associations caused by LD with a known disease locus. The method is especially suited for datasets of multi-allelic markers, and does not require assumptions about the disease model. The procedure can be adapted for tests using unphased genotypes or TDT-like tests.

\section{Acknowledgements}

The Type 1 diabetes data was made available by Dr. John Todd. We thank Dr. Frank Dudbridge for his help with the CETDT, and the anonymous reviewers for their constructive comments on the manuscript. This research was supported by a grant of the Netherlands Organization for Scientific Research (NWO). Information on the Type 1 Diabetes dataset is available at: http://www-gene.cimr.cam.ac.uk/todd. The UNPHASED package is available at the website of the UK Human Genome Mapping Project Resource Centre (HGMP):

http://www.hgmp.mrc.ac.uk/ fdudbrid/. CYGWIN and GCC are available from: http://www.cygwin.com.

\section{References}

Bain, S.C., Todd, J. A. \& Barnett, A.H. (1990) The British Diabetic Association-Warren repository. Autoimmunity 7 , $83-5$.

Beck, S. \& Trowsdale, J. (2000) The human major histocompatibility complex: lessons from the DNA sequence. Annu Rev Genet 1, 117-37.
Clerget-Darpoux, F., Babron, M.C., Prum, B., Lathrop, G.M., Deschamps, I. \& Hors, J. (1988) A new method to test genetic models in HLA associated diseases: the MASC method. Ann Hum Genet 52, 247-258.

Cordell, H.J. \& Clayton, D.G. (2002) A unified stepwise regression procedure for evaluating the relative effects of polymorphisms within a gene using case/control or family data: application to HLA in type 1 diabetes. Am J Hum Genet 70, 124-41.

Dudbridge, F. (2003) Pedigree disequilibrium tests for multilocus haplotypes. Genet Epidemiol 25, 115-21

Ewens, W.J. \& Spielman, R.S. (1995) The transmission/disequilibrium test: history, subdivision and admixture. Am J Hum Genet 57, 455-464.

Falk, C. \& Rubinstein, P. (1987) Haplotype relative risks: an easy reliable way to construct a proper control sample for risk calculations. Ann Hum Genet 51, 227-33.

Hedrick, P.W. (1987) Gametic disequilibrium measures: proceed with caution. Genetics 117, 331-41.

Herr, M., Dudbridge, F., Zavattari, P., Cucca, F., Guja, C., March, R., Campbell, R.D., Barnett, A.H., Bain, S.C., Todd, J.A. \& Koeleman, B.P. (2000) Evaluation of fine mapping strategies for a multifactorial disease locus: systematic linkage and association analysis of IDDM1 in the HLA region on chromosome 6p21. Hum Mol Genet 9, 1291-301.

Koeleman, B.P., Dudbridge, F., Cordell, H.J. \& Todd, J.A. (2000a) Adaptation of the extended transmission/disequilibrium test to distinguish disease associations of multiple loci: the Conditional Extended Transmission/Disequilibrium Test. Ann Hum Genet 64, 207-13.

Koeleman, B.P., Herr, M.H., Zavattari, P., Dudbridge, F., March, R., Campbell, D., Barnett, A.H., Bain, S.C., Mulargia, A.P., Loddo, M., Amos, W., Cucca, F. \& Todd, J.A. (2000b) Conditional ETDT analysis of the human leukocyte antigen region in type 1 diabetes. Ann Hum Genet 64, 215-21.

Li H (2001) A permutation procedure for the haplotype method for identification of disease-predisposing variants. Ann Hum Genet 65, 189-96.

Lie, B.A., Todd, J.A., Pociot, F., Nerup, J., Akselsen, H.E., Joner, G., Dahl-Jorgensen, K., Ronningen, K.S., Thorsby, E. \& Undlien, D.E. (1999) The predisposition to type 1 diabetes linked to the human leukocyte antigen complex includes at least one non-class II gene. Am J Hum Genet. 64, 793-800.

Moghaddam, P.H., Zwinderman, A.H., de Knijff, P., Roep, B.O., Schipper, R.F., Van der Auwera, B., Naipal, A., Gorus, F., Schuit, F. \& Giphart, M.J. (1997) TNFa microsatellite polymorphism modulates the risk of IDDM in Caucasians with the high-risk genotype HLA DQA1 $1^{0501}$-DQB1 $1^{0201} / \mathrm{DQA1} 1^{0301}$-DQB1 ${ }^{0302}$. Diabetes 46, 1514-5. 
Robinson, W.P., Barbosa, J., Rich, S.S \& Thomson, G. et al. (1993) Homozygous parent affected sib pair method for detecting disease predisposing variants: application to insulin dependent diabetes mellitus. Genet Epidemiol 10, 273-88.

Rubio, J.P., Bahlo, M., Butzkueven, H., van der Mei, I.A.F., Sale, M.M., Dickinson, J.L., Groom, P. et al. (2002) Genetic dissection of the Human Leukocyte Antigen region by use of haplotypes of Tasmanians with Multiple Sclerosis. Am J Hum Genet 70, 1125-1137.

Sham, P.C. \& Curtis, D. (1995) An extended transmission/disequilibrium test (TDT) for multi-allele marker loci. Ann Hum Genet 59, 323-36.
Valdes, A.M. \& Thomson, G. (1997) Detecting diseasepredisposing variants: the haplotype method. Am J Hum Genet 60, 703-16.

Valdes, A.M., Noble, J.A., Genin, E., Clerget-Darpoux, F, Erlich, H.A. \& Thomson, G. (2001) Modeling of HLA class II susceptibility to Type I diabetes reveals an effect associated with DPB1. Genet Epidemiol 21, 212-23.

Received: 31 October 2003

Accepted: 04 August 2004 